\title{
Binary Contention Resolution for M2M Random Access Prioritization in LTE-A and 5G
}

\author{
Mikhail Vilgelm, Wolfgang Kellerer \\ Chair of Communication Networks \\ Technical University of Munich, Germany \\ Email: \{mikhail.vilgelm, wolfgang.kellerer\}@tum.de
}

\begin{abstract}
Accommodating Machine-to-Machine (M2M) applications and their requirements is one of the challenges on the way from LTE towards 5G networks. A notorious bottleneck for supporting M2M in LTE is the Random Access (RA) procedure for network connection establishment. To address the limitations of LTE RA, we introduce here the idea of Binary Contention Resolution (BCR), a simple and efficient method for prioritizing the devices during RA. Preliminary simulative evaluation shows the gains of using BCR in comparison to state-of-the-art.
\end{abstract}

\section{INTRODUCTION}

Accommodating Machine-to-Machine (M2M) communication is one of the important requirements for the evolution from LTE-Advanced towards the new generation $5 \mathrm{G}$ networks. In particular, key challenges for existing LTE-A networks are supporting diverse M2M performance requirements, and accommodating large number of M2M User Equipments (UEs) [1], [2]. The latter challenge leads to the necessity for re-designing the LTE-A radio resource management procedures. That is, since M2M UEs are expected to communicate infrequently, radio bearer maintenance for every UE is not possible. Hence, Random Access (RA) procedure has to precede every M2M transmission for establishing the connection between UE and a Base Station (BS) [3]. RA procedure is notorious for high delay and high drop probabilities in the case of high load [2], because of the contention-based nature of the protocol.

Important challenge of the RA procedure is to provide quality of service (QoS) differentiation. Standard LTE-A supports QoS by the means of Access Class Barring (ACB). It relies on the BS broadcasting barring factor $p_{b}^{i}$ for every QoS class $i$, which is used by every UE to probabilistically decide if it attempts RA procedure or backs off. Many recent studies have proposed improvements for ACB, with dynamic adjustment of $p_{b}^{i}$, or tweaking other contention parameters (back-offs, duty cycles, etc.) [3]-[5]. However, prioritization using contention parameters suffers from major drawbacks: it does not provide sufficient isolation between classes (i.e., UEs from different classes still collide), and it relies on the RA load estimation, which might be unreliable [3].

In this paper, we introduce a Binary Contention Resolution (BCR) approach for supporting QoS in LTE-A RA (inspired by the protocol used in powerline communication (PLC), bus, and ad-hoc networks [6]). BCR is enabled by the modified slot structure for connection request (MSG3, see Figs. 1a,b). In the contrast to the state-of-the-art, BCR does not require load estimation, and is independent of the contention. We introduce the idea in Sec. II-B, compare performance with $\mathrm{ACB}$ in Sec. II-C, and discuss implementation challenges and further work in Sec. III.

ISBN 978-3-901882-94-4 (c) 2017 IFIP (a)

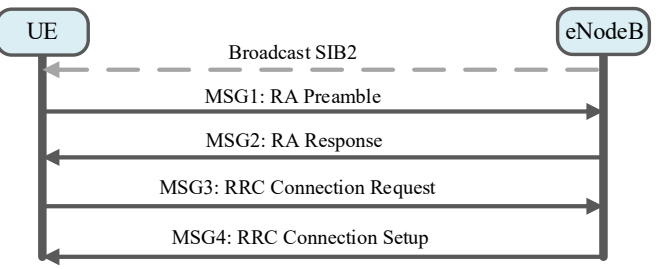

(b)

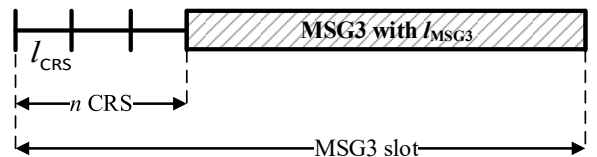

Fig. 1: (a) Standard protocol for LTE Random Access Procedure. (b) Proposed RRC Connection Request (MSG3) slot structure for Binary Contention Resolution.

\section{RANDOM ACCESS PROCEDURE AND BINARY CONTENTION RESOLUTION}

\section{A. Conventional Random Access Procedure in LTE-A}

Random Access Procedure starts with UE retrieving Physical Random Access Channel configuration from the BS broadcast (see Fig. 1a). The configuration contains contention parameters (preamble sets, back-off, barring probability, etc.), and the location of the next RA slot. UE proceeds with sending a randomly chosen preamble (codeword) as MSG1 in the RA slot, and receives MSG2 as a BS reply, containing the timing and location of the sub-frame for MSG3 for every received ("activated") preamble. Note that a BS is only able to detect if a particular preamble has been activated, but not how many UEs have sent it. Hence, if two or more UEs choose the same preamble at MSG1, their MSG3s collide. If connection requests collide, no MSG4 is received from the $\mathrm{BS}$, and the collided UEs will re-attempt sending the preambles after a random back-off time. For brevity, we omit further details of RA procedure which can be found in [3]-[5].

\section{B. Proposed Binary Contention Resolution (BCR) Protocol}

Since UEs collisions happen while sending Connection Request (MSG3), our proposed scheme is leaving preamble sequence and preamble response unmodified, and focuses directly on MSG3. All UEs activating the same preamble during MSG1 are allocated the same uplink resources for the transmission of their RRC Connection Requests. However, in BCR, we propose to precede MSG3 transmission with $n$ contention resolution slots (CRSs, see Fig. 1b): these slots are used by UEs to transmit a binary level sequence: every CRS is either $L$ or 0 (presence or absence of a signal), depending on the priority class of a given UE. With $n$ CRS up to $k=2^{n}$ priority classes are possible, and the binary sequence for a given priority class $i$ is computed as $s_{i}=[k-1-i]_{2}$ (higher priority class corresponds to the larger binary number $s$ ). All involved UEs are listening to CRSs, and, if they detect the 
presence of a higher priority UE, they do not send MSG3, and go directly for the back-off.

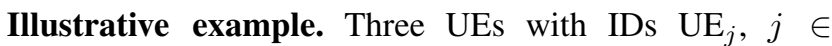
$\{1,2,3\}$, from priority classes 0,1 and 2 , respectively, are choosing the same preamble for MSG1, and, hence, are allocated same uplink slot for MSG3. Given $n=2$ priority resolution slots, binary sequences for UEs are $s_{\mathrm{UE}_{1}}=\{L, L\}$, $s_{\mathrm{UE}_{2}}=\{L, 0\}$, and $s_{\mathrm{UE}_{3}}=\{0, L\}$ respectively. In the first CRS, only $\mathrm{UE}_{1}$ and $\mathrm{UE}_{2}$ are transmitting signal, while $\mathrm{UE}_{3}$ is listening. Since $\mathrm{UE}_{3}$ is detecting a non-empty signal in the first CRS, it concludes that higher priority UE is present, and, hence, drops the attempt and goes for back-off. At the second CRS, only $\mathrm{UE}_{1}$ sends the signal, and $\mathrm{UE}_{2}$ is listening. Since the signal from $\mathrm{UE}_{1}$ is present, $\mathrm{UE}_{2}$ does not proceed with transmission. As a result, $\mathrm{UE}_{1}$ wins the contention and continues with RRC Connection Request transmission.

\section{Performance Comparison: BCR vs. $A C B$}

For evaluation, we consider a scenario of temporary RA channel overload: semi-simultaneous arrival of a "burst" of connection requests from a large number of UEs (e.g., UEs reconnecting after a blackout, or emergency situation reporting by redundant sensors) [7]. Every UE $j \in[1, N]$ in the cell is activated within total activation time $T_{A}$ with Beta-distributed individual activation time $t_{a}^{i}$ [4], [5]:

$$
g\left(t_{a}^{j}\right)=\frac{\left(t_{a}^{j}\right)^{\alpha-1}\left(T_{A}-t_{a}^{j}\right)^{\beta-1}}{T_{A}^{\alpha+\beta-2} \mathcal{B}(\alpha, \beta)}, \quad 0 \leq t_{a}^{j} \leq T_{A} .
$$

In Fig. 2, we compare the results of prioritization of two classes ("prio" and "non-prio") for BCR and ACB, with respect to two metrics: (a) average serving time (time until a UE is successfully connected to BS) $\bar{t}_{s}=\sum_{j=1}^{N} t_{s}^{j}$, and (b) effective throughput $T$, defined as the ratio of successful UEs and total amount of available resources. For BCR, we set a fixed barring probability $p_{b}^{1}=p_{b}^{2}=0.5$. To ensure fair comparison, we set ACB parameters $p_{b}^{1}, p_{b}^{2}$ such that $\bar{t}_{s}^{\text {prio }}(\mathrm{ACB})=\bar{t}_{s}^{\text {prio }}(\mathrm{BCR})$, i.e., class "prio" has the same serving time for both schemes. From Fig. 2(a) we observe that ACB scheme deteriorates the performance of the non-prio class significantly, and BCR results in up to 4 times lower delay for non-prio class. From Fig. 2(b), we also observe that BCR achieves $0.2-10$ times throughput increase, compared to ACB.

\section{OUtLOOK: PRACTICAL CONSIDERATIONS AND Challenges}

Although we have shown that BCR achieves lower delays and has better resource utilization than state-of-the-art ACB, there are several prerequisites for the practical implementation of BCR. They are listed here with a brief discussion, and have to be comprehensively addresses by the future work on BCR.

Power control. First of all, BCR relies on "overhearing" the signal from other UEs. That means that UEs are required to listen to the medium in MSG3 slot, and that UEs' power levels have to be sufficient to reach all contenders. This is a typical case for relatively small and dense cells, but might not be true for large cells, e.g., in a rural area. Henceforth, impact of the transmission power on the BCR performance is non-negligible and has to be evaluated.

Protocol overhead. Given the MSG3 duration as $l_{\mathrm{MSG} 3}$, desired number of QoS classes $k$, and CRS slot duration as

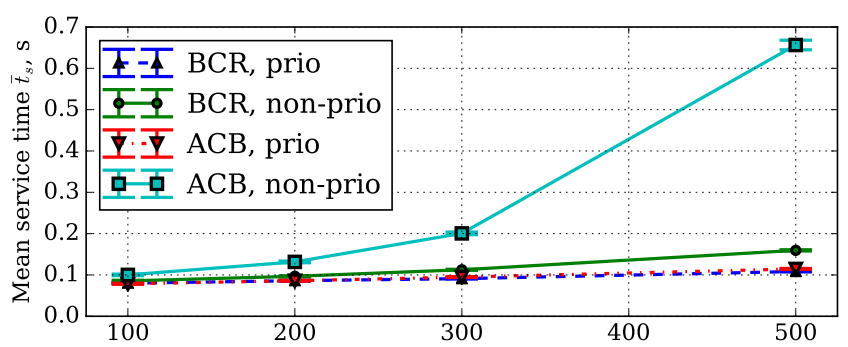

(a) Number of UEs $N$

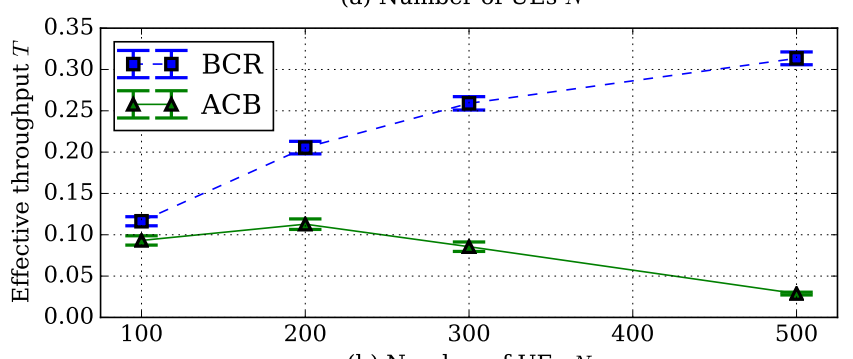

(b) Number of UEs $N$

Fig. 2: (a) Mean serving time and (b) Effective throughput for $T_{A}=0.1 \mathrm{~s}, \alpha=3$, $\beta=4$. Barring probabilities (BCR) $p_{b}^{1}=p_{b}^{2}=0.5$, number of preambles $M=54$. Average values over 30 runs with $95 \%$ confidence intervals.

$l_{\mathrm{CRS}}$, the signaling overhead of $\mathrm{BCR}$ is:

$$
x=1+\frac{l_{\mathrm{CRS}}\left\lceil\log _{2} k\right\rceil}{l_{\mathrm{MSG} 3}},
$$

where $\left\lceil\log _{2} k\right\rceil=n$ is the required number of CRS slots $n$ to accommodate $k$ QoS classes. If MSG3 is relatively small, the CRS slot length $l_{\mathrm{CRS}}$ strongly influences the overhead. This length is determined by the physical layer, and has to be properly chosen to ensure reliable detection (in PLC IEEE $\left.1901 l_{\mathrm{CRS}} \approx 36 \mu \mathrm{s}[6]\right)$.

Additionally, future work on BCR includes analytical modeling, and applications to persistent RA overload. Also, it is clear that the proposed scheme only eliminates the collision between different priority classes, while the UEs of the same priority can still collide. Hence, combinations of BCR with recently proposed techniques for optimizing preamble contention, e.g., distributed queuing [2], tree algorithms [4], or dynamic ACB [5], need to be investigated.

\section{REFERENCES}

[1] A. Osseiran et al., "Scenarios for 5G mobile and wireless communications: the vision of the METIS project," IEEE Communications Magazine, vol. 52, no. 5, pp. 26-35, 2014.

[2] A. Laya, L. Alonso, and J. Alonso-Zarate, "Is the random access channel of LTE and LTE-A suitable for M2M communications? A survey of alternatives," IEEE Comm. Surveys \& Tutorials, 2014.

[3] M. Vilgelm, H. M. Gürsu, W. Kellerer, and M. Reisslein, "LATMAPA: Load-Adaptive Throughput-MAximizing Preamble Allocation for Prioritization in 5G Random Access," IEEE Access, January 2017.

[4] H. M. Gürsu, M. Vilgelm, W. Kellerer, and M. Reisslein, "Hybrid Collision Avoidance-Tree Resolution for M2M Random Access," IEEE Trans. on Aerospace and Electronic Systems, vol. PP, no. 99, 2017.

[5] S. Duan, V. Shah-Mansouri, Z. Wang, and V. Wong, "D-ACB: Adaptive congestion control algorithm for bursty M2M traffic in LTE networks," IEEE Trans. Vehicular Technology, vol. PP, no. 99, pp. 1-1, 2016.

[6] T. Gehrsitz, R. Durner, H. Kellermann, H.-T. Lim, and W. Kellerer, "Priority-based energy-efficient MAC protocols for the in-car power line communication," in IEEE VNC, Proc. of. IEEE, 2014, pp. 61-68.

[7] 3GPP, "Technical Report 37.868: Study on RAN Improvements for Machine-type Communications (Release 11)," 3GPP Technical Specification Group Radio Access Network, Tech. Rep., 2011. 